\title{
A Novel Miniature Mixing Device For Polymeric Blends and Nanocomposites
}

\author{
Martin Sentmanat, ${ }^{1}$ Christos Stamboulides, ${ }^{2}$ Savvas G. Hatzikiriakos ${ }^{2}$ \\ ${ }^{1}$ Xpansion Instruments, 1425 Glenoak Dr., Tallmadge, Ohio 44278 \\ ${ }^{2}$ Department of Chemical and Biological Engineering, The University of British Columbia, Vancouver, \\ BC V6T-1Z4, Canada
}

\begin{abstract}
A new miniature mixer has been developed to monitor and optimize the preparation protocol of various polymeric compounds and blend systems. The effect of mixing time and other basic processing parameters on the shear and extensional rheological properties of said compounds and blends is examined to understand the effect of undermixed and/or overmixed conditions on the rheological properties and thus the quality of the final products. Results from the new miniature mixer are compared with the results from other conventional mixing techniques to assess the scalability of the new mixing protocol. Two examples are used, those of polymer blending and nanocomposite formation. POLYM. ENG. SCl., 49:2092-2098, 2009. ( 2009 Society of Plastics Engineers
\end{abstract}

\section{INTRODUCTION}

Blends of linear and branched polymers are used extensively in the polymer processing industry to optimize melt processing behavior as well as final product properties. In practice, mixing of the polymer blend constituents typically takes place in an internal batch mixer or extruder depending on the chemical, macrostructural, and rheological behaviors of the blend components [1, 2]. Efficient blending is crucial for the quality of the final polymeric blend products. Nanocomposites and in particular dispersion of nanoclays into polymeric matrices to create intercalated and exfoliated morphologies is another case where efficient mixing is needed [3-7]. Similarly, internal batch mixers or extruders are used to perform rigorous mixing to attain intercalated and/or exfoliated morphologies [3-5, 7].

Automated internal batch mixer devices with a container or enclosed mixing vessel/chamber have been in use since the 19th century for the preparation and compounding of foodstuffs, clays, cements, plastics, and other fluid-like

Correspondence to: Martin Sentmanat; e-mail: martin@xpansioninstruments. com

DOI 10.1002/pen.21447

Published online in Wiley InterScience (www.interscience.wiley.com). (C) 2009 Society of Plastics Engineers media. Although the art of automated mixing technology may have its roots from over a century ago, the science of automated mixing has slowly evolved over just the past few decades [2]. Regardless of the size or scale of the processing operation whether for the production of bread dough or the compounding of complex polymeric materials, the ability of individual material components to intimately interact in a controlled manner is a fundamental principle of all material mixing and compounding operations.

Small laboratory-scale mixers were developed for mixing protocol evaluation purposes from a quality control and research and development capacity to study, develop, and qualify new materials and compounds, improve industrial-scale mixing efficiencies and predictability, and to reduce the cost and time associated with industrialscale pilot mixing trials. Although many commercially available laboratory-scale mixer devices currently exist, few can handle batch capacities less than $5 \mathrm{~mL}$ in total volume. This is particularly important when exotic experimental filler materials such as nanoclays or model polymers are available or synthesized in small quantities.

In this article, a novel miniature mixing device that enables polymer mixing and compounding on the sub- $5 \mathrm{~mL}$ scale from temperatures ranging from subambient to the melt state was used to compound blends of linear low-density polyethylenes (LLDPE) and low-density polyethylenes (LDPE) polymer melts and to form exfoliated polymeric nanocomposites. Subsequent to mixing, rheological results for LLDPE/LDPE blends and polymeric nanocomposites compared favorably with data for the same materials compounded with a conventional laboratory-scale internal batch mixer (Haake Rheomix 3000), a twin screw extruder (Micro 27, Leistritz) and a Killion single screw extruder in conjuction with a grinder as illustrated by the linear viscoelastic and extensional flow properties.

\section{The XIM Miniature Mixer}

The new Xpansion Instruments Mini-Mixer (XIM) device is a miniature, detachable dual rotor/chamber 


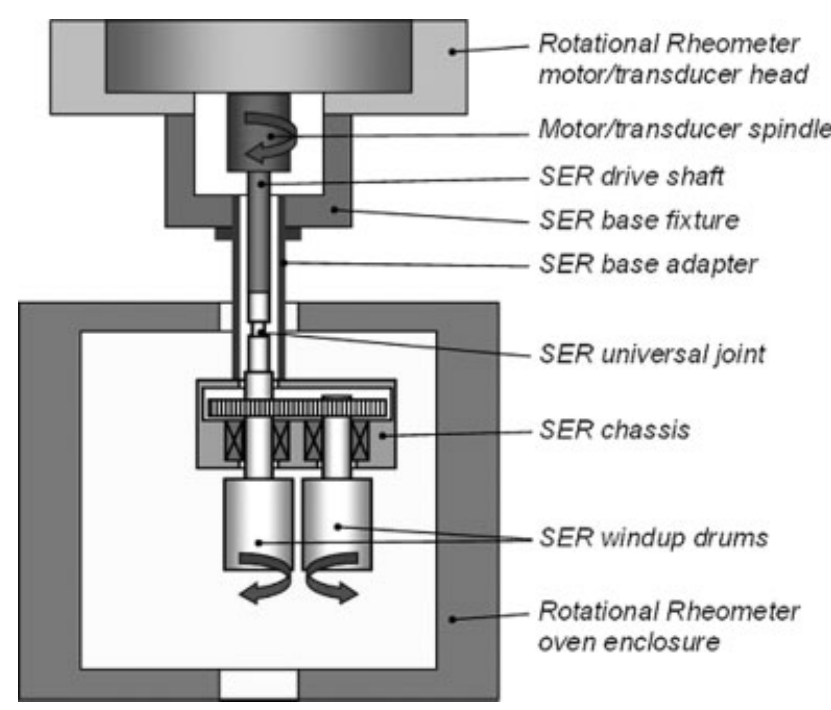

FIG. 1. Schematic of the SER2 extensional rheometer.

mixer attachment for use on the sentmanat extensional rheometer 2 (SER2) model line of the SER Universal Testing Platform from Xpansion Instruments [8, 9]. The XIM can be accommodated within the oven chamber of the host rheometer system and/or can be accommodated with independent band heater control for controlled temperature mixing evaluation.

Figure 1 shows a schematic of the new SER2 design that is used to perform extensional rheological measurements as well as a number of other physical property characterizations. This miniature physical material characterization device has been designed as a detachable fixture for use on a number of commercially available torsional rotational rheometers. Its unique design allows the precision rotational motions of the rotational rheometer to be translated into precision linear motions when the ends of a sample are secured to each windup drum. Connected internally by intermeshing gears, the rotation of the master drum drives the counter rotation of the slave drum thereby uniformly stretching the attached sample across the web spanning the drums. Designed to be accommodated within the convection oven chamber of the commercial rotational rheometer, a variety of physical material properties can be characterized as a function of temperature. When hosted on a single transducer/motor rotational rheometer, the windup drums of the SER2 can also be submerged within a controlled temperature fluid environment thereby allowing for physical properties characterization under fluid immersion testing. Furthermore, the windup drums of the SER2 have been designed to be detachable and replaceable to allow for a variety of drum surface treatments or finishes that can enhance sample gripping characteristics or that can be tailored for the characterization of surface friction, abrasion, and adhesion properties of sample materials.

Utilizing these fluid immersion and detachable drum capabilities of the SER2, the XIM is a miniature dual rotor internal batch mixer that has been designed as an attachment to the SER2 model line as depicted in Fig. 2. With the windup drums removed, the mixing rotors of the XIM insert into the drive hubs of the SER2 to facilitate rotor detachment and replacement during mixing compound removal. The XIM mixing chamber has a duallobed cavity with a length of $16.3 \mathrm{~mm}$ and a lobe radius of $6.9 \mathrm{~mm}$ with a total evacuated chamber capacity of $5 \mathrm{~mL}$. The XIM comes standard with two different dual rotor sets with a nominal rotor radius of $6.7 \mathrm{~mm}$, a 2wing rotor configuration that allows for a maximum allowable mixer capacity of $3.75 \mathrm{~mL}$, and a sigma blade rotor configuration that allows for a maximum allowable mixer capacity of $4 \mathrm{~mL}$. The XIM has been designed to be accommodated within the convection oven chamber of a number of commercial rotational rheometers but can also be accommodated with an electrical resistance band
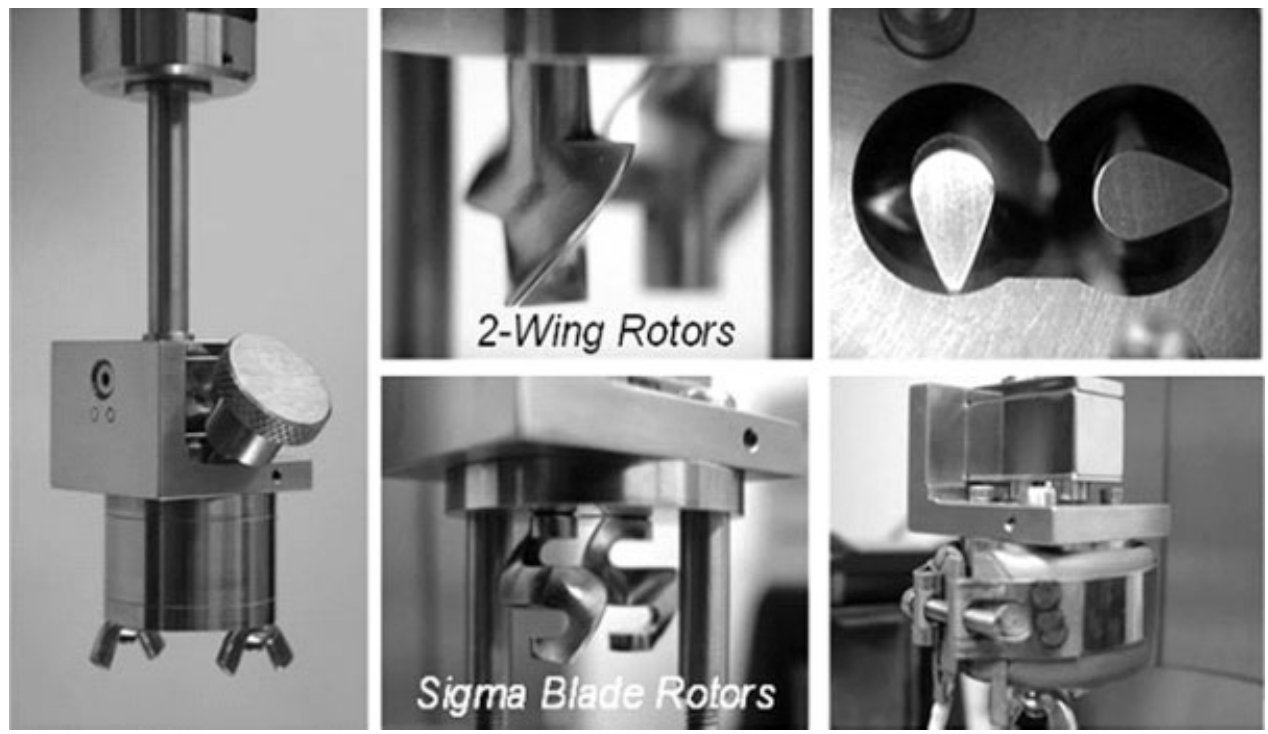

FIG. 2. Photos of the XIM mixer with different types of mixing elements and heater options. 
heater for independent temperature control during mixing. In this manner, the SER2 when configured with the XIM miniature mixer attachment can allow a commercial torsional rotational rheometer to serve as a miniature internal batch mixer capable of mixing, compounding and blending of dough, pastes, gels, and polymer melts on a 2$2.5 \mathrm{~g}$ batch mixing capacity while monitoring the realtime torque evolution for mixing protocol evaluation.

\section{MATERIALS AND METHODS}

Four different polymers were used to prepare the polymeric blends. Two LLDPEs, namely a Ziegler-Natta hexane-copolymer, Z-N LLDPE-I (LL3001) and a metallocene butane-copolymer m-LLDPE II (Exact 3128 ) both supplied by ExxonMobil and two LDPEs, the LDPE-I (EF606A) provided by Westlake Polymers and the LDPE-II (662I) provided by Dow Chemicals. Table 1 lists all the polymers used along with their melt indices, densities at room temperature and their zero-shear viscosities at $150^{\circ} \mathrm{C}$ that provide indications of their relative molecular weights. The zero-shear viscosities were determined by creep testing in an Anton Paar MCR 501 rotational rheometer using a $25 \mathrm{~mm}$ diameter parallel plate geometry. A constant shear stress of $10 \mathrm{~Pa}$ was used to attain very low shear rates to reach the Newtonian viscosity flow regime.

Blends were prepared at 50 wt $\%$ using three methods; by means of a Killion single screw extruder using a screw with mixing elements at a speed of $20 \mathrm{rpm}$ and temperature of $160^{\circ} \mathrm{C}$, a Haake Rheomix 3000 configured with dual 2wing rotors at $160^{\circ} \mathrm{C}$ and the new XIM mini-mixer (described in the previous section) attached to a model SER2-P hosted on an Anton Paar MCR 501 rotational rheometer and configured with the dual 2-wing rotors at $210^{\circ} \mathrm{C}$ - the elevated mixing temperature with the XIM was required to ensure that the mixing torques from both polymer blends fell within the upper torque limit $(0.2 \mathrm{~N}$ $\mathrm{m})$ of the host rotational rheometer system. The polyethylene ZN-LLDPE-I was mixed with the LDPE-I at $50 \mathrm{wt} \%$ to produce an immiscible blend [10] and m-LLDPE was mixed with LDPE-II at $50 \mathrm{wt} \%$ to produce a miscible blend [11]. The former blend will be referred to as polymer blend-I from now on (less viscous) and the latter will be referred to as polymer blend-II (more viscous).

The quality of mixing was examined by rheological measurements. It has been reported that rheological properties (mainly extensional) are extremely sensitive to

TABLE 1. Properties of polyethylene resins used in this study.

\begin{tabular}{llccr}
\hline Sample ID & Resin type & $\begin{array}{c}\text { Melt index } \\
(\mathrm{g} / 10 \mathrm{~min}) \\
\left(190^{\circ} \mathrm{C}\right)\end{array}$ & $\begin{array}{c}\text { Density } \\
(\mathrm{g} / \mathrm{cc}) \\
\left(25^{\circ} \mathrm{C}\right)\end{array}$ & \multicolumn{1}{c}{$\begin{array}{c}\eta_{\mathrm{o}} \\
(\mathrm{Pa} \cdot \mathrm{s}) \\
150^{\circ} \mathrm{C}\end{array}$} \\
\hline ZN-LLDPE- I & LL3001 & 1.0 & 0.917 & 17,488 \\
m-LLDPE II & Exact 3128 & 1.3 & 0.900 & 11,383 \\
LDPE-I & EF606A & 2.2 & 0.919 & 44,234 \\
LDPE-II & Dow 662I & 0.47 & 0.919 & 127,900 \\
\hline
\end{tabular}

levels of long chain branching [10-13] and it is manifested by a certain degree of strain hardening [9]. An appropriate mixing/distribution of the branches of LDPE into the LLDPE matrix will be reflected as a comparative agreement of the rheological properties of blends prepared by the various mixing methods.

The nanocomposites were formed with the use of Fusabond EMB-226DE, a commercially available chemically modified polyethylene from DuPont. Fusabond is functionalized by maleic anhydride grafted to help bond together polymers used in toughened, filled and blended compounds. It increases the filler acceptability of the polymers by a good margin. The maleic anhydride graft level in the Fusabond used is said to be $0.89 \%$ and the typical density is $0.93 \mathrm{~g} / \mathrm{cc}$ at $23^{\circ} \mathrm{C}$. The melt flow rate at $190^{\circ} \mathrm{C} / 2.16 \mathrm{~kg}$ is $1.82 \mathrm{dg} / \mathrm{min}$ and the melting point is about $120^{\circ} \mathrm{C}$. Nanomer I.44 P, from Nanocor, is used in this study as the organically surface modified montmorillonite clay. Montmorillonite is a 2:1 phyllosilicate wherein tetrahedral sheets of silica are fused with octahedral sheets of aluminum. The aspect ratios of montmorillonites are as high as 1000 for individual platelets and 300-500 for tactoids with an interlamellar space or gallery of about $1 \mathrm{~nm}$ separating the platelets. Thus structural asymmetry of montmorillonites combined with a large surface area of about $700-800 \mathrm{~m}^{2} / \mathrm{g}$ and a high cation exchange capacity (CEC) of about 70-150 meq/100 g render this class of silicates open to intercalation $[6,7]$.

\section{Preparation of Blends}

Single Screw Extrusion. The LDPE resin was melt blended respectively with the corresponding LLDPE resin at a weight composition of 50/50. The blending with the single screw method was performed as follows: the original components were mixed and grinded in a Brabender mixer/grinder to reduce their pellet size. Then, the mixture in the form of flakes was manually blended and fed into a single screw extruder at a processing speed of about $20 \mathrm{rpm}$, using a screw having mixing elements near to the end of the metering zone. The temperature of the die was kept constant at about $160^{\circ} \mathrm{C}$. The extrudates were then pelletized for easy handling. Manual mixing of flakes before feeding into the single screw extruder significantly helps to produce well mixed blends $[6,7]$.

Haake Rheomix 3000. The blends were prepared by processing the pellets of the two polymers first dry blended at the right proportion into the chamber of the Haake Rheomix 3000 internal mixer with two counter rotating roller rotors at a velocity ratio of $2 / 3$. The geometrical parameters of the dual-lobed cavity mixing chamber were $34 \mathrm{~mm}$ in radius and $82 \mathrm{~mm}$ in length (nominal mixer capacity of $300 \mathrm{~mL}$ ). The chamber was loaded to $70 \%$ of its total volume for all batches, for a total of about $210 \mathrm{~mL}$ for both of the blends. The polymers were blended at $160^{\circ} \mathrm{C}$ using a constant rotational 

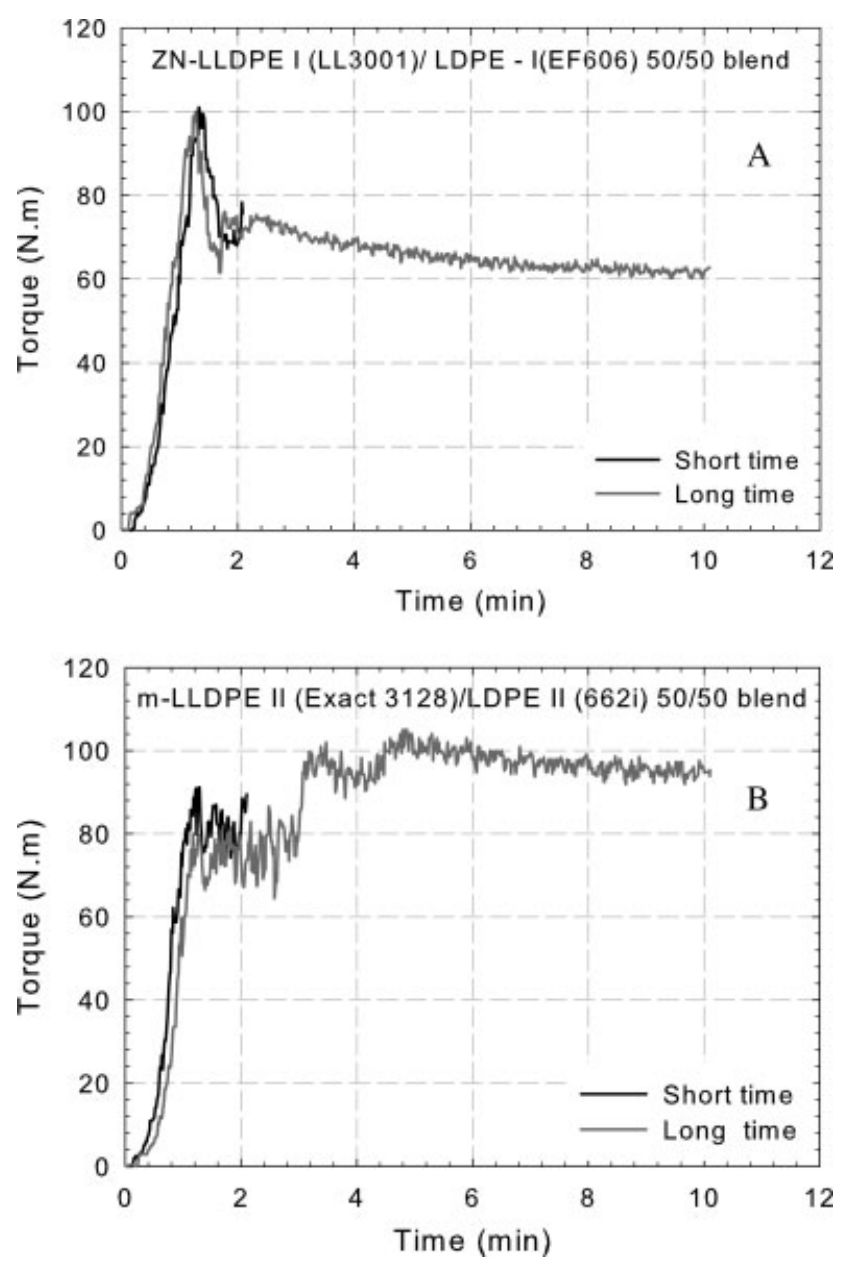

FIG. 3. (a) The torque curve for blend ZN-LDPE-I (LL3001)/LDPE-I (EF606) (Blend I) prepared with the Haake Rheomix 3000 at $50 \mathrm{rpm}$ and $160^{\circ} \mathrm{C}$. (b) The torque curve for blend m-LDPE-II (Exact3128)/ LDPE-II (662i) (Blend II) prepared with the Haake Rheomix 3000 at $50 \mathrm{rpm}$ and $160^{\circ} \mathrm{C}$.

speed of $50 \mathrm{rpm}$ for short times (when the torque just overcomes the maximum) and for long times (when the torque approaches a steady-state value).

Figure $3 \mathrm{a}$ and $\mathrm{b}$ shows the torque curves for the two blends. In both cases about $8-10 \mathrm{~min}$ are needed to approach steady-state, although a maximum appears for the less viscous blend. The runs referred to as short times were stopped after 2 min with the objective of obtaining under-mixed blends (incomplete mixing), however, in both the short and long time mix cases, at the cutoff time a maximum mixing torque had already been achieved and as such no appreciable difference was observed in the rheological behaviors between any of the short and long mix time polymer blends.

XIM Mixing. The corresponding torque mixing curves for the two polymer blends obtained with the XIM miniature mixer in the rotational rheometer at $210^{\circ} \mathrm{C}$, are plotted in Fig. 4. Despite a rotor speed of only $10 \mathrm{rpm}$, the mixing appears to be more intensive in nature with the $\mathrm{XIM}$ and hence complete mixing is achieved in signifi-

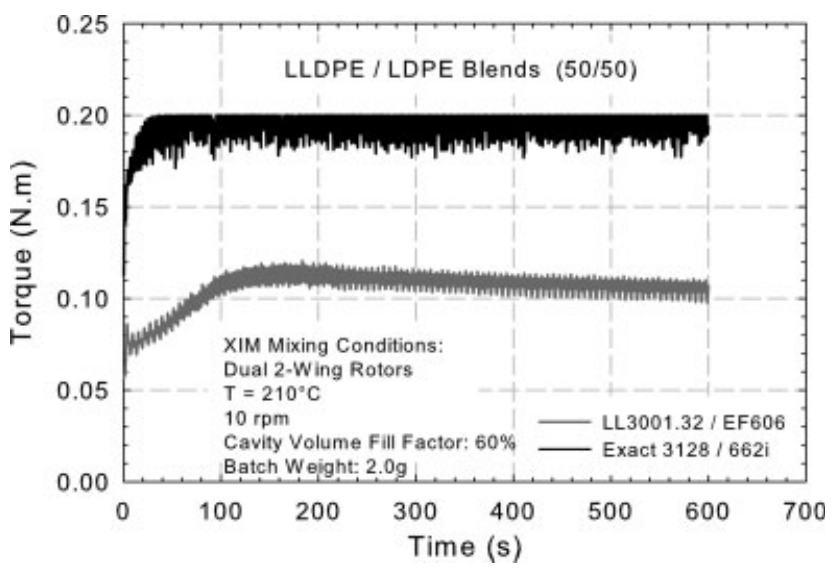

FIG. 4. The torque curve for blends I and II with XIM at $10 \mathrm{rpm}$ and $210^{\circ} \mathrm{C}$.

cantly shorter periods of time compared to those obtained in the Haake Rheomix 3000. A mixing temperature of $210^{\circ} \mathrm{C}$ with the XIM was chosen for both polymer blends to ensure that the mixing torques from the higher viscosity polymer blend-II fell within the upper torque limit $(0.2 \mathrm{~N} \mathrm{~m})$ of the host rotational rheometer system. The ratio of the steady-state torques for the two blends in the Haake Rheomix and XIM are about the same indicating relative consistency between the two blending operations. The chamber of the XIM was loaded up to $80 \%$ of its maximum volumetric capacity $(3.75 \mathrm{~mL})$ yielding a total batch weight of $2.0 \mathrm{~g}$ for each polymer blend.

\section{Preparation of Nanocomposites}

Twin Screw Extruder Mixing. Two different nanocomposite compounds were prepared at nanoclay filler loading levels of 2 wt $\%$ and 4 wt \% within a matrix of Fusabond. The raw materials were mixed together and fed through a single feeder into a corotating twin screw extruder-Micro 27, Leistritz, Germany (L/D = 36). A screw diameter of $27 \mathrm{~mm}$ and a screw rotational speed of

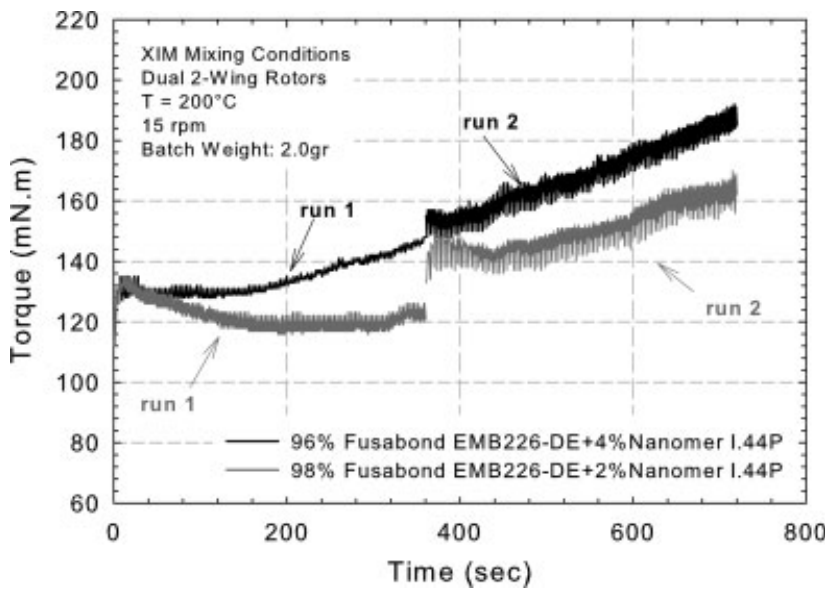

FIG. 5. The torque curve for nanocomposites with Fusabond and 2 and 4 wt \% nanoclays with XIM at $15 \mathrm{rpm}$ and $200^{\circ} \mathrm{C}$. 


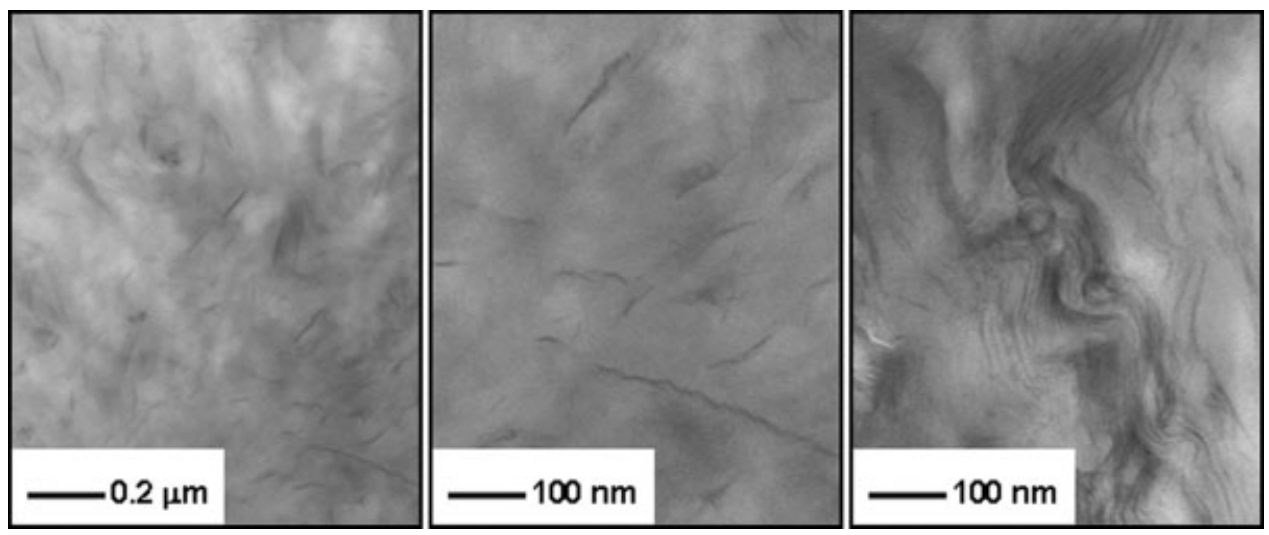

FIG. 6. TEM images of $96 \%$ Fusabond compound with $4 \%$ clay at different magnifications (see scales in each image). Figure $6 \mathrm{a}$ and $\mathrm{b}$ show mostly exfoliated morphologies, whereas Fig. $6 \mathrm{c}$ shown intercalated morphology.

$200 \mathrm{rpm}$ were used to generate a mass flow rate of $10 \mathrm{~kg}$ / h. The barrel was programmed at 190, 200, 210, 210, $200,190,190$, and $190^{\circ} \mathrm{C}$ and was equipped with vacuum degassing. The extruded strands were water cooled over a distance of $100 \mathrm{~cm}$ and were strand pelletized. The extruded pellets were melt-pressed into thin sheets of $2 \mathrm{~mm}$ thickness in a Carver hydraulic press at $150^{\circ} \mathrm{C}$ and specimen of desired dimensions were cut out of the sheet: $25 \mathrm{~mm}$ diameter for rheological testing in a parallel plate shear geometry with the Anton Paar MCR 501 rotational rheometer. It was observed during melt pressing that with increasing incorporation of Fusabond, the material adheres strongly to the polyester sheet used to compression mold the compound. However, a reduction of interfacial adhesion between the molded sample and the polyester film was observed with increasing nanoclay filler composition, an indication of how the nanoclay appears to serve as a mold release agent. This observation could be related to the effect reported by Hatzikiriakos et al., [6] that organically modified nanoclays at small concentrations well-dispersed in a polyethylene matrix act as processing aids, eliminating instabilities such as melt fracture.

XIM Mixing. The mixing in the XIM miniature mixing device was performed at $210^{\circ} \mathrm{C}$ and a rotational speed of $15 \mathrm{rpm}$ for a total batch weight of $2.0 \mathrm{~g}$ for each Fusabond/ nanoclay composite. The torque mixing curves for the two nanocomposites obtained with the XIM mixing in the rotational rheometer are plotted in Fig. 5. It appears that the torque keeps increasing in a continuous fashion which is attributed to the continuous formation of intercalated and possibly exfoliated morphologies. The mixing was stopped after 6 min (run 1) and it was continued (run 2) after a few hours of polymer relaxation. Upon reinitiation of mixing, the torque curves appear to continue from the previous torque level achieved before the termination of the first mixing stage. With continued mixing, it appears as though the torque has not attained a steady-state value even after a total mixing duration of $12 \mathrm{~min}$, although the curve for the 2 wt $\%$ dispersion appears to approach a steady-steady value after 12 min of mixing. The mixing duration for both nanocomposite compounds was terminated after $12 \mathrm{~min}$ to ensure that the mixing torque for the $4 \mathrm{wt} \%$ nanocomposite compound remained within upper torque limit $(0.2 \mathrm{~N} \mathrm{~m})$ of the host rotational rheometer system.

Figure 6 illustrates a well-distributed matrix with further dilution of clay for the $4 \mathrm{wt} \%$ nanoclay compound. The presence of intercalated morphologies in some areas (Fig. 6c) and exfoliated morphologies in some other areas (Fig. 6a and b) can be observed providing an indication of the mixing efficiency that can be achieved with the XIM miniature mixing device in forming intercalated and exfoliated morphologies with nanocomposite compounds. Such morphologies inherently require rigorous shear and extensional mixing to be formed.

\section{Rheological Measurements}

Polymer Blends. Figures 7 and 8 depict the linear viscoelastic measurements obtained on samples mixed using the Killion single screw extruder, the Haake Rheomix 3000

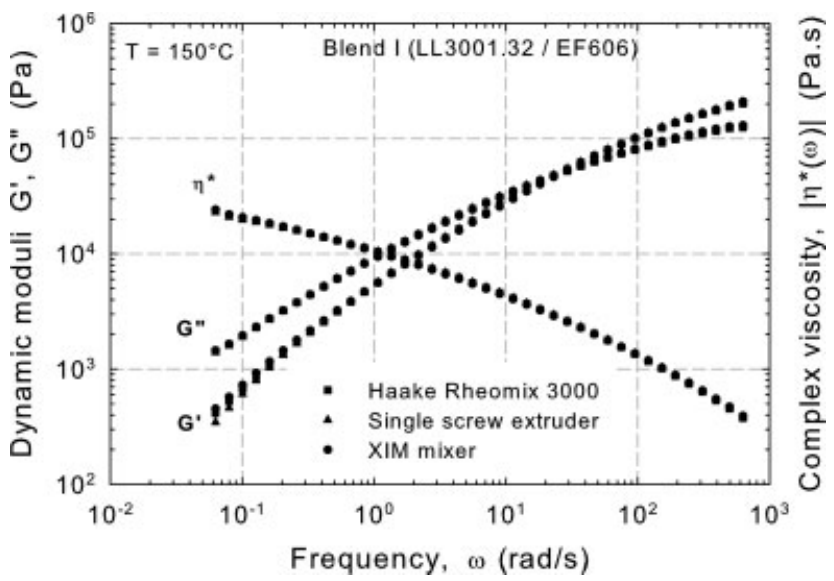

FIG. 7. Linear viscoelastic moduli, $\mathrm{G}^{\prime}, \mathrm{G}^{\prime \prime}$, and complex viscosity, $\left|\eta^{*}(\omega)\right|$ of blend I prepared by single screw extrusion, Haake Rheomix 3000 and the XIM mixer. 


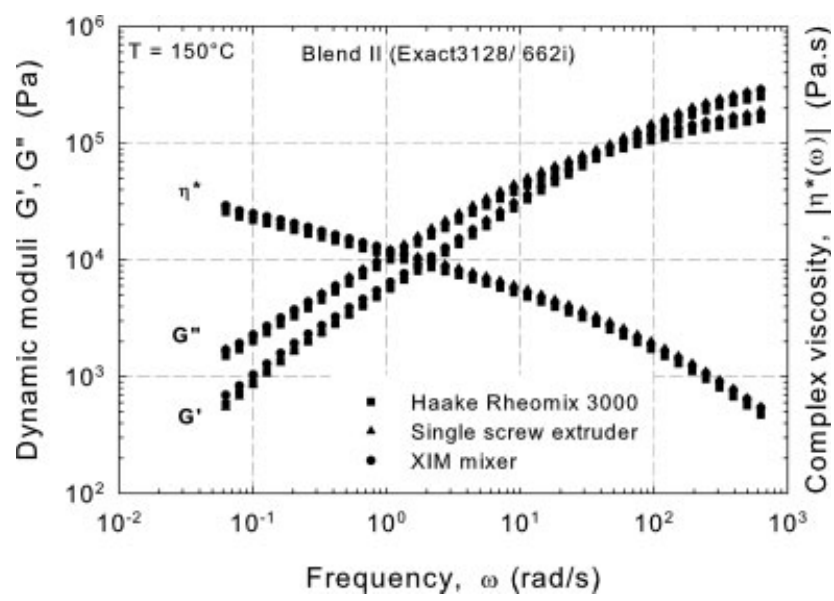

FIG. 8. Linear viscoelastic moduli, $\mathrm{G}^{\prime}, \mathrm{G}^{\prime \prime}$, and complex viscosity, $\left|\eta^{*}(\omega)\right|$ of blend II prepared by single screw extrusion, Haake Rheomix 3000 and the XIM mixer.

and the XIM mini-mixer for the two polymer blends respectively measured at $150^{\circ} \mathrm{C}$ in small amplitude oscillatory shear with $25 \mathrm{~mm}$ diameter parallel plates on the Anton Paar MCR 501. The superposition of the rheological data is notable thereby providing a comparative illustration of the mixing consistency achieved with all three blending methods. In particular the excellent agreement of $G$ ' provides a strong indication of the mixing consistency achieved with the XIM mini-mixer despite being more than two orders of magnitude smaller in capacity than the other two blending devices.

A stronger indication of excellent mixing can be elucidated by comparing the extensional rheological behavior of the polymer blends. Extensional rheology is a sensitive tool in detecting subtle changes in polymer macrostructure, particularly with regard to long chain branching [13, 14]. Transient extensional rheological measurements were performed on all of the polymer blends with the model SER2-P hosted on an Anton Paar MCR 501 rotational

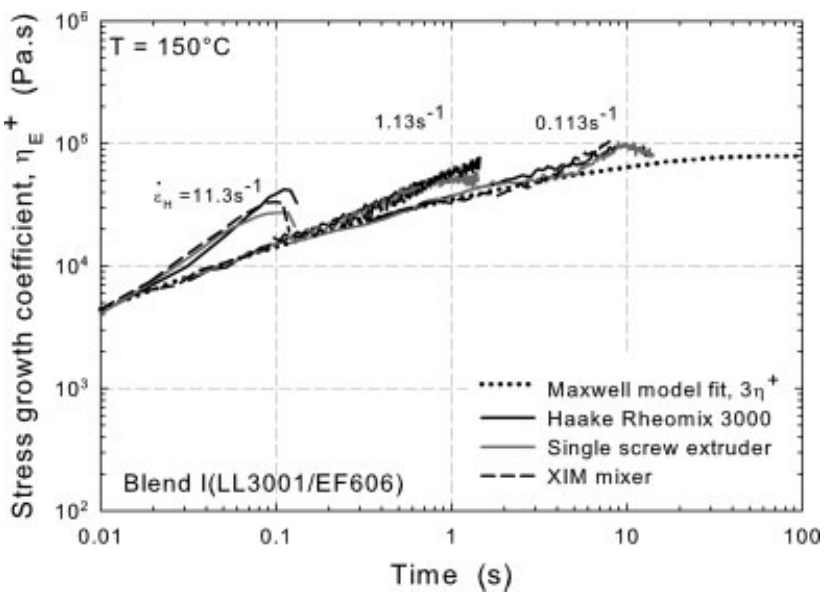

FIG. 9. The tensile stress growth coefficient, $\eta_{E}^{+}$, of blend I prepared by single screw extrusion, Haake Rheomix 3000 and the XIM mixer, at three different Hencky strain rates: $0.113,1.13$, and $11.3 \mathrm{~s}^{-1}$ at $150^{\circ} \mathrm{C}$.

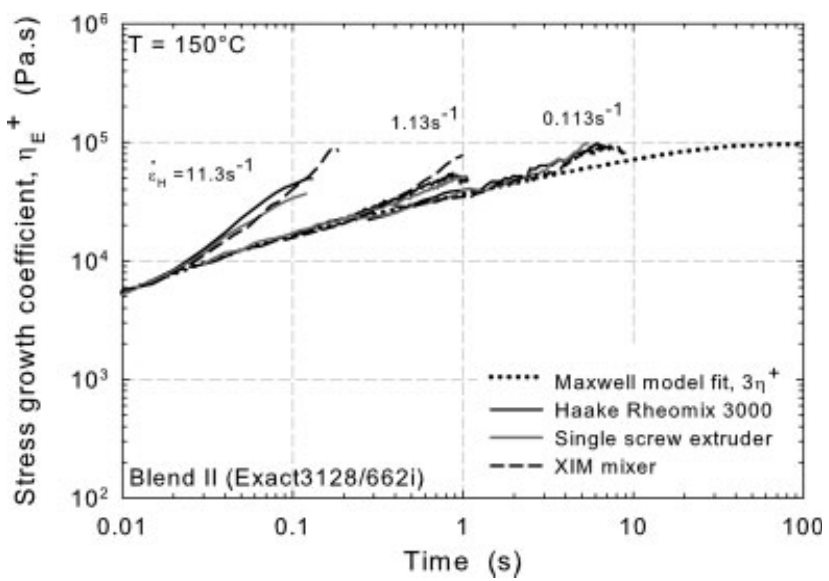

FIG. 10. The tensile stress growth coefficient of blend II prepared by single screw extrusion, Haake Rheomix 3000 and the XIM mixer, at three different Hencky strain rates: $0.113,1.13$, and $11.3 \mathrm{~s}^{-1}$ at $150^{\circ} \mathrm{C}$.

rheometer system. Figures 9 and 10 compare the tensile stress growth coefficient of the blends prepared by using the Killion single screw extruder, the Haake Rheomix 3000 and the XIM mini-mixer for the two polymer blends respectively at three Hencky strain rates at a test temperature of $150^{\circ} \mathrm{C}$. The agreement is remarkable indicating the excellent similarity of blend properties attained with all three methods of mixing. The degree and onset of strain hardening for all the blends at the three Hencky strain rates exhibit excellent agreement demonstrating that structurally all of the polymer blends prepared by the different methods are essentially the same. The extensional viscositities of the pure components of the blends have been previously reported in refs $[10,11]$ and are not plotted in Figures 9 and 10 for the sake of clarity.

Polymer Nanocomposites. Figures 11 and 12 plot the complex viscosity, $\left|\eta^{*}(\omega)\right|$ of pure Fusabond EMB226 and Fusabond with 2 wt $\%$ and 4 wt $\%$ nanoclays correspond-

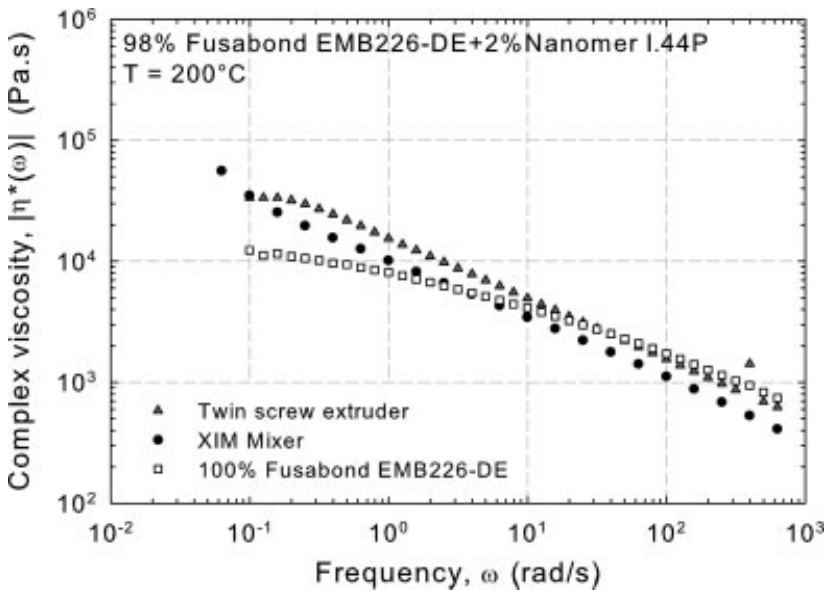

FIG. 11. The complex viscosity, $\left|\eta^{*}(\omega)\right|$ of pure Fusabond EMB226 and Fusabond with 2 wt \% nanoclays prepared by twin screw extrusion, Micro 27, Leistritz and the XIM mixer. 


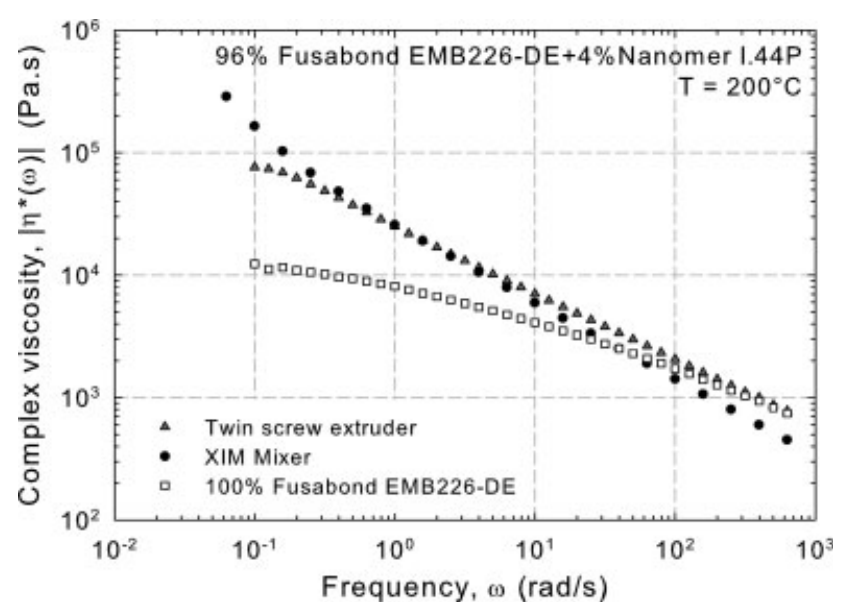

FIG. 12. The complex viscosity, $\left|\eta^{*}(\omega)\right|$ of pure Fusabond EMB226 and Fusabond with 4 wt \% nanoclays prepared by twin screw extrusion, Micro 27, Leistritz and the XIM mixer.

ingly prepared by twin screw extrusion (Micro 27, Leistritz) and the XIM mini-mixer. First, the significant increase in viscosity of the nanocomposites is due to intercalated and exfoliated morphology (see TEM images in Fig. 6). The agreement in the rheology of the compouds prepared by the two methods is reasonable (better for the $4 \mathrm{wt} \%$ compounds) considering the sensitivity of the rheology on the degree of intercalation and exfoliation $[3,5,7]$. Note that the compounds prepared with the XIM mini-mixer indicate vertically asymptotic behavior at small frequencies suggesting the existence of a yield stress-behavior that is typically observed for well-dispersed filled polymer compounds [14]. These results indicate that the degree of exfoliation in the nanocomposites compounded with the XIM mini-mixer is higher than the degree of exfoliation achieved with the twin screw extruder, a result that is somewhat surprising.

\section{CONCLUSIONS}

A novel miniature internal batch mixing device, the XIM, was developed for use in blending and compounding materials in $2.0 \mathrm{~g}$ batch sizes. Rheological results on materials mixed with the XIM superpose with data for materials compounded on mixing devices more than two orders of magnitude larger in scale, namely a Haake Rheomix 300 and a Killion single screw extruder and a twin screw extruder (Micro 27, Leistritz). These results demonstrate that materials can be mixed and compounded at very small batch sizes with the XIM miniature mixer and have similar physical property characteristics with materials prepared with conventional compounding operations. In addition the results for these and other blends, suggest that this novel commercial mixing device can be successfully used as an invaluable miniature-scale laboratory tool for predicting mixing and compounding protocols before large scale implementation. Finally, where small quantities of materials are available (specialized model polymer samples and exotic filler materials), this device is an invaluable tool to study blending and compounding.

\section{REFERENCES}

1. L.A. Utracki, Polymer Alloys and Blends, Hanser, New York (1990).

2. C. Rauwendaal, Polymer Mixing, A self-Study Guide, Hanser, New York (1998).

3. G. Galgali, "Synthesis-Structure-Processing-Property Relationships in Polymer Nanocomposites," Ph.D. Thesis, National Chemical Laboratory, India (2003).

4. E.P. Giannelis, Adv. Mater., 8, 29 (1996).

5. T.G. Gopakumar, J.A. Lee, M. Kontopoulou, and J.S. Parent, Polymer, 43, 5483 (2002).

6. S.G. Hatzikiriakos, N. Rathod, and E.B. Muliawan, Polym. Eng. Sci., 45, 1098 (2005).

7. R. Devendra, S.G. Hatzikiriakos, and R. Vogel, J. Rheol., 50, 415 (2006).

8. M. Sentmanat, U.S. Patent 6,578,413 (2003).

9. M. Sentmanat, Rheol. Acta, 43, 657 (2004).

10. O. Delgadillo-Velázquez, S.G. Hatzikiriakos, and M. Sentmanat, Rheol. Acta, 47, 19 (2008a).

11. O. Delgadillo-Velázquez, S.G. Hatzikiriakos, and M. Sentmanat, J. Polym. Sci. Part B Polym. Phys., 46, 1699 (2008b).

12. S.G. Hatzikiriakos, Polym. Eng. Sci., 40, 2279 (2000).

13. M. Sentmanat, E.B. Muliawan, and S.G Hatzikiriakos, Rheol. Acta, 44, 1 (2005).

14. J.M. Dealy and K.F. Wissbrun, Melt Rheology and its Role in Plastics Processing. Theory and Applications, Van Nostrand Reinhold, New York (1990). 